\title{
Fundamentals of the Densification Mechanism of NiCuZn-Ferrites
}

\author{
D.HOLZ $^{\text {a) }}$, A. PAGANA ${ }^{\text {b) }}$, V. TSAKALOUDI ${ }^{\text {b) }}$, G. KOGIAS ${ }^{\text {b), c) }}$ and V. ZASPALIS ${ }^{\text {b), c) }}$
}

a) Ferroxcube Polska / YAGEO Corporation, ul. Zwierzyniecka 2, Skierniewice 96-100, Poland

b) Laboratory of Inorganic Materials, Chemical Process and Energy Resources Institute, Centre for Research and Technology-Hellas CERTH, $6^{\text {th }} \mathrm{km}$. Charilaou-Thermi Rd, Thermi-Thessaloniki 57001, Greece

c) Laboratory of Materials Technology, Department of Chemical Engineering, Aristotle University of Thessaloniki, 54124 Thessaloniki, Greece

\begin{abstract}
The partial substitution of $\mathrm{Ni}$ with $\mathrm{Cu}$ in NiZn-ferrites has many economical advantages in bulk magnetic component manufacturing and additional densification advantages in multilayer chip inductor manufacturing. However, the detailed mechanism through which the presence of $\mathrm{Cu}$ influences the densification of NiZn-ferrites has not been outlined in literature. In this article the mechanism of $\mathrm{NiCuZn-densification} \mathrm{has} \mathrm{been} \mathrm{explained} \mathrm{on} \mathrm{basis} \mathrm{of} \mathrm{point} \mathrm{defect} \mathrm{chemistry} \mathrm{and} \mathrm{changes} \mathrm{in} \mathrm{the} \mathrm{type} \mathrm{of} \mathrm{major} \mathrm{defects} \mathrm{that} \mathrm{dominate} \mathrm{ion}$ transport through sintering. Although the densification of NiZn-ferrites is mainly dictated by the $\mathrm{Fe}$ content and is restricted at Fe excess, in the presence of $\mathrm{Cu}$ this limitation does not hold any more because of the additional anion vacancies introduced due to $\mathrm{Cu}^{2+}$ to $\mathrm{Cu}^{+}$ reduction at elevated temperatures. In addition, further $\mathrm{Cu}$ reduction, through the usage of reductive atmospheres, enhances the densification even further and this provides an extra processing parameter for tuning densification. There is no evidence for liquid phase sintering due to the formation of liquid phases ought to the presence of $\mathrm{Cu}$ or associated eutectic mixtures.
\end{abstract}

Key Words: NiCuZn ferrite; densification; anion vacancies; point defect chemistry

\section{INTRODUCTION}

NiZn ferrites are among the most widely used soft magnetic materials for high frequency applications due to their high electrical resistivity and low losses [1, 2]. Many of the essential properties (e.g. mechanical, electrical, optical, magnetic properties etc), strongly depend, besides on the basic chemical composition and the morphology of the microstructure, on the relative density of the polycrystalline system [3]. In general, high densities favor a good application performance. The final density is acquired at the final heating stage where the actual sintering process takes place, during which there is mass transfer to the vacant space and an increase in density occurs. In the case of ionic solids such as the magnetic ceramic materials of the chemical formula $\left(\mathrm{Ni}_{x} \mathrm{Cu}_{y} \mathrm{Zn}_{1-x-y}\right) \mathrm{Fe}_{2+\delta} \mathrm{O}_{4}$, mass transfer during the intermediate and final sintering stages takes place through ion diffusion via point defects such as vacancies in the lattice [4]. The aim of this study is to understand the detailed mechanism through which the presence of $\mathrm{Cu}$ influences the densification of NiZn-ferrites during compositional (Fe and $\mathrm{Cu}$ content) and operational (partial oxygen pressure) changes in order to be able to synthesize high quality NiCuZn-ferrites.

\section{EXPERIMENTAL PROCEDURE}

Ceramic materials of the chemical formula $\left(\mathrm{Ni}_{\mathrm{x}} \mathrm{Cu}_{y} \mathrm{Zn}_{0.494}\right) \mathrm{Fe}_{2+\delta} \mathrm{O}_{4}[(x+\mathrm{y}=0.494),(y=0,0.106,0.206),(\delta=-0.05,-0.03$, $-0.01,0,0.02,0.05)]$ were prepared by solid state synthesis, as reported elsewhere [4]. Uniaxially compacted rods of 3.00 $\mathrm{g} / \mathrm{cm}^{3}$ green density were used for thermomechanical analysis (Dilatometer $402 \mathrm{PC}$, Netzsch) at $1200^{\circ} \mathrm{C}$ under a heating rate of $5^{\circ} \mathrm{C} \min ^{-1}$, in air, $\mathrm{N}_{2}$ or mixed $40 \% \mathrm{O}_{2}-60 \% \mathrm{~N}_{2}$ flows.

\section{RESULTS AND DISCUSSION}

The shrinkage at $1050^{\circ} \mathrm{C}$ for $\mathrm{NiCuZn}$-ferrites with different iron and copper contents and different partial pressures of oxygen in the sintering atmosphere are shown in Figure 1. It has been considered that densification at this stage is dominated by the ion diffusion and any differences in the final densities can be traced-back in differences in the diffusion coefficients which in turn are determined by the concentrations of point defects. This consideration is found to be in agreement with the experimental results.

As shown in Figure 1 (curve 1), the stoichiometric and no copper containing material exhibits a densification minimum approximately at stoichiometry. This can be explained by the fact that at stoichiometry the concentration of point defects is 
minimum. At iron concentrations lower or higher than approximately $x=2$ the densification increases due to the creation of defects such as oxygen vacancies, cation interstitials or divalent cation vacancies, respectively.

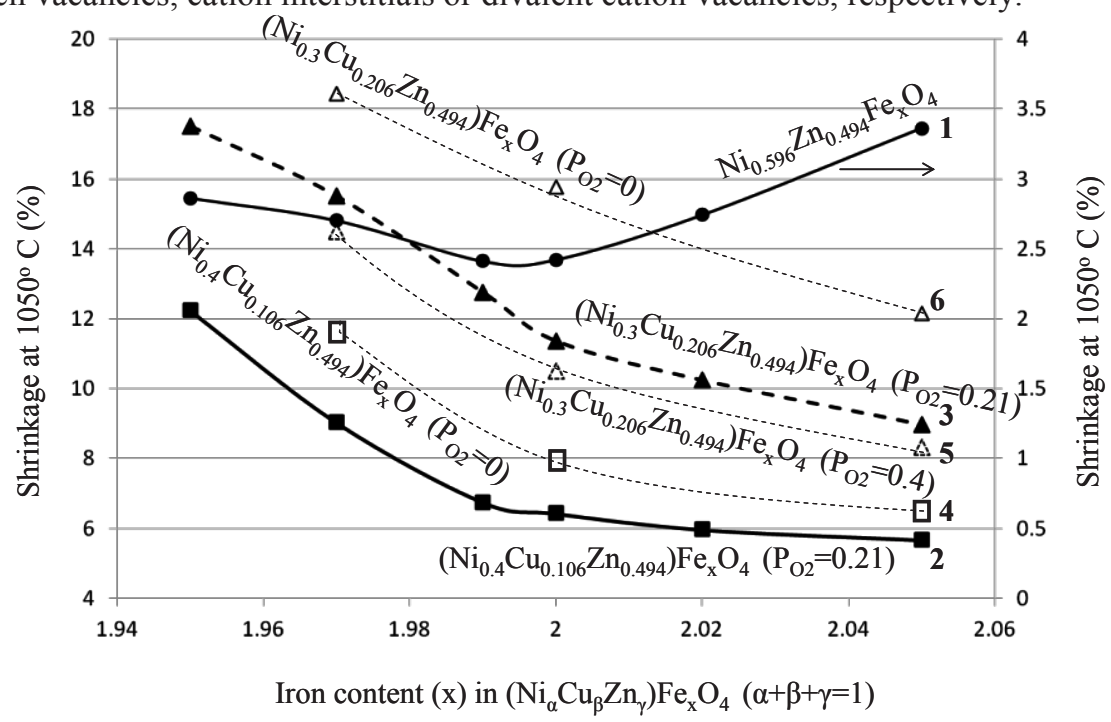

Fig. 1 Shrinkage as a function of iron content for specimens with different copper contents and sintered under different partial pressures of oxygen.

This behavior is almost independent of the oxygen partial pressure since the temperature of $1050^{\circ} \mathrm{C}$ is considered low for significant iron reduction. By constructing a simple defect model according to which an excess of $(1+\delta) \mathrm{mole}$ of $\mathrm{Fe}_{2} \mathrm{O}_{3}$ or an excess of $\left(1+\delta^{\prime}\right)$ mole of divalent $\mathrm{AO}$ oxide react to form a spinel structure with 1 mole $\mathrm{AO}$ or $\mathrm{Fe}_{2} \mathrm{O}_{3}$ respectively, the percentage of $\left[\mathrm{V}_{\ddot{\mathrm{O}}}\right]$ or $\left[\mathrm{V}_{\dot{\mathrm{A}}}^{\prime}\right]$ defects as a function of $\delta$ or $\delta$ ' can be calculated to be [5]:

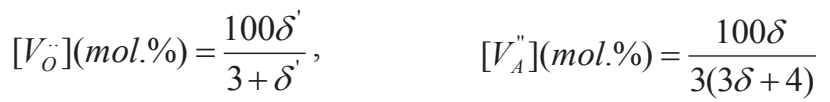

Equations (1) are plotted in Figure 2 (curve 1) and it can be seen that there is qualitative agreement with the experimental results of Figure 1 (by comparing curves (1) of both Figures). When $\mathrm{Cu}$ is inserted into the material as substitution for $\mathrm{Ni}$, the minimum at stoichiometry is not detected (Figure 1, curves 2 and 3 ). This is because at elevated temperatures a reduction of copper takes place according to the equilibrium:

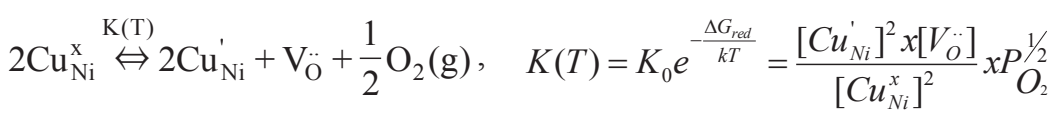

This reduction of copper creates negatively charged sites for the compensation of which oxygen vacancies are assumed to be generated. Thus at stoichiometry, there is no change of the dominating defect type and oxygen vacancies continues to be the dominating defect type even under iron excess conditions. If we denote y the fraction of copper ions that are reduced (which depends on the value of the equilibrium constant and the partial pressure oxygen), then equations (1) can be modified to be:

$$
x>2, \quad\left[\mathrm{~V}_{\ddot{\mathrm{O}}}\right](\mathrm{mol} . \%)=\frac{200 \mathrm{y}}{3 \delta+4} ; \quad x<2, \quad\left[V_{\ddot{\circ}}\right](\text { mol. } \%)=\frac{100\left(2 \delta^{\prime}+3 y\right)}{8\left(3+\delta^{\prime}\right)}
$$

For arbitrary selected values of $y=10,50$ and $70 \%$ the percentage of oxygen vacancies as a function of the iron content $\mathrm{x}$ is shown in curves 2,3 and 4 of Fig. 2, in good qualitative agreement with the experimental curves 2 and 3 of Fig. 1. 


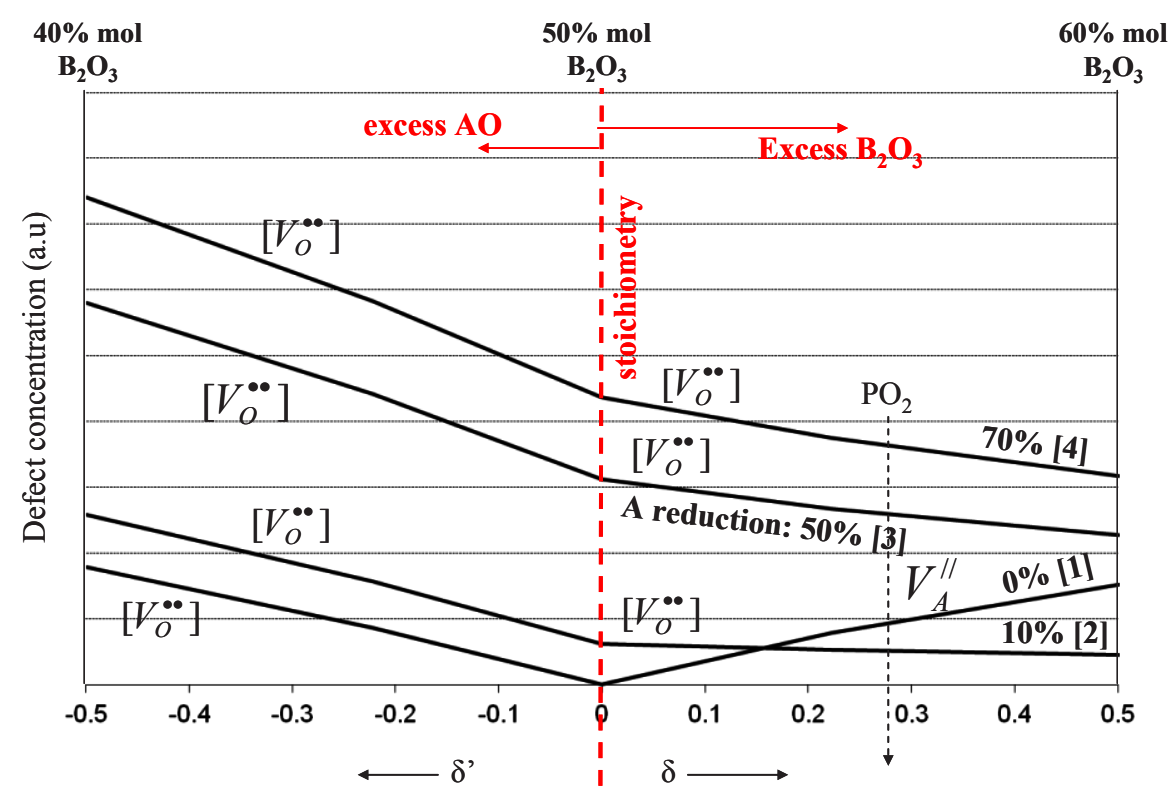

Fig. 2 Simplified point defect concentrations as a function of stoichiometry in the absence and in the presence of copper that is being reduced during sintering.

Since in the presence of copper the dominating type of defects remain the oxygen vacancies even in the cation deficient (or $\mathrm{Fe}_{2} \mathrm{O}_{3}$ excess) region the partial pressure of oxygen is expected to influence the densification (through y or through equilibrium equation (2)). This explains the experimentally found dependency of the densification on the partial pressure of oxygen only in the presence of copper (by comparing curves 2 and 4 or curves 3,5 and 6 of Figure 1). Worthwhile mentioning is that the higher the concentration of copper in the $\mathrm{Fe}_{2} \mathrm{O}_{3}$-excess material, the higher the concentration of oxygen vacancies and therefore also the higher the influence of the oxygen partial pressure on densification. Although the presence of cation interstitials as copper reduction compensating defects cannot be excluded, the qualitative agreement between the experimental results and the model prediction suggests that densification is dominantly controlled by the larger slowest diffusing oxygen anions through anion vacancies.

\section{CONCLUSIONS}

The effect of copper on the densification of ( $\mathrm{NiCuZn}$ )-ferrites has been investigated. Based on the assumption that densification is governed by ionic diffusion the results could be qualitatively explained through a simplified defect model according to which the presence and subsequent reduction of copper preserves the same type of defect (oxygen vacancies) at iron contents below or above stoichiometry. Besides accelerating densification because of oxygen vacancy concentration enhancement, the presence of copper offers the extra possibility for further densification enhancement through the usage of low partial pressures of oxygen. The above-presented model constituted the basis for the development of improved NiCuZn ferrite materials of high density and excellent magnetic properties under either Fe-excess or deficiency. Additionally, it is an indication that the mechanism through which the addition of $\mathrm{Cu}$ enhances densification does not include liquid phase sintering, but solely the creation of vacancies and acceleration of diffusion.

\section{REFERENCES}

[1] A. Globus, R.V. Monjaras, IEEE Trans. Magn. 11, 1300 (1975).

[2] A. Goldman, “Modern Ferrite Technology”, Van Nostrand Reinhold, New York, 1990, p.145.

[3] W. D. Kingery, H. K. Bowen, and D. R. Uhlmann, "Introduction to Ceramics", Wiley, New York, 1976, p.448.

[4] D. Sakellari, V. Tsakaloudi, E.K. Polychroniadis and V. Zaspalis, J. Am. Ceram. Soc. 91, 366 (2008).

[5] D. M. Smyth, "The defect chemistry of metal oxides", Oxford University Press, 2000, p. 17. 